age. Treatments that incorporate self-regulation such as autogenic thermal feedback training require an understanding of an internal control mechanism. From a medical perspective the foundation for self-management and self-regulation of behavior develops as the child applies autonony to managing the external world. Awareness of autonomy, initiative and industry issues may facilitate successful treatment of childhood headache. A shift in family attention to headache free days could be a strong treatment reinforcer for children, focusing less attention on the headaches. (Marcon RA, Labbe EE. Assessment and treatment of children's headaches from a developmental perspective. Headache Sept $1990 ; 30: 586-592)$.

COMMENT. Adding a developmental perspective to psychological interventions in the management of childhood headache is likely to increase treatment effectiveness. The management of headache in children is different from that of adults and requires an assessment of cognitive and affective development and a knowledge of children's concepts of illness and pain. Factors in precipitating headaches such as diet and exercise may be more important in children than in adults and will require special attention. The use of long-term investigational medications in children is undesirable and relaxation techniques and dietary modification may be nore effective and appropriate.

\title{
PATHOPHYSIOLOGY OF VASCULAR HEAD PAIN
}

The mechanism of head pain, provocative stimuli, and receptors involved in drug treatment are reviewed from the Stroke Research Laboratory, Neurosurgery and Neurology, Massachusetts General Hospital, Boston, MA. A primitive defensive fiber network surrounds the brain and its blood vessels which is derived principally from trigeminal ganglia neurons. Headaches develop from activity within the trigeminal vascular system and are controlled by serotonin agonists that couple with 5-HT receptors on unmyelinated $\mathrm{C}$ fibers to block activity in the trigeminovascular system. Neuropeptide release and neurogenic inflammation within the coverings of the brain are inhibited. Altered physiologic states (stress, fatigue), bright lights and sleep deprivation may precipitate migraine and act by modulating neuronal activity and synaptic events within cortical gray matter. Local increases in cortical blood flow modulated by trigeminovascular fibers facilitate removal of potentially noxious chemicals. (Moskowitz MAA. The visceral organ brain: Implications for the pathophysiology of vascular head

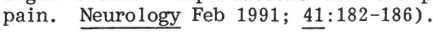

OOMMENT. That vasospasm contributes to monosodium glutamate-induced headache has been demonstrated by Merritt JE and Williams PB in the Department of Pharmacology, Eastern Virginia Medical School, Norfolk, VA. (Headache Sept 1990; $30: 575-580)$. The direct effects of glutamate and its metabolite glutamine on arterial contractility were examined using rings of rabbit aorta. High concentrations of glutamate caused significant concentration dependent contractions. At high concentrations 
the calcium channel blocker Verapamil inhibited the glutamate response. Monosodium glutamate has a direct effect on peripheral arterial tone and may represent a serious health hazard to certain individuals wi th vascular disease.

A study of treatments used by patients for migraine prior to attending the City of London Migraine $\mathrm{Clinic}$ showed that alternative medical treatments were popular including acupuncture, hypnotherapy, homeopathy, feverfew, osteopathy, yoga, and vitamin supplements. The authors emphasize the importance of an accurate history of all medications used, whether prescribed or over-the-counter. (MacGregor EA et al. Headache Sept 1990; $30: 571-574$ ).

\section{MUSCLE DISORDERS}

\section{PREDNISONE IN DUAHENNE DYSTROPHY}

The results of a randamized controlled trial of daily Prednisone conducted in 99 boys aged five to 15 years wi th Duchenne dystrophy are reported from the Department of Neurology, University of Rochester, NY and five collaborating institutions. Prednisone at $0.3 \mathrm{mg} / \mathrm{kg}$ or 0.75 $\mathrm{mg} / \mathrm{kg}$ and placebo were administered for six months. Patients were evaluated at ten days, one month, two months, three months, and six months of treatment. At the three month visit those receiving 0.75 $\mathrm{mg} / \mathrm{kg}$ were significantly strongly than those given $0.3 \mathrm{mg} / \mathrm{kg}$ Prednisone and both Prednisone groups were stronger than those treated with placebo. At six months side-effects occurred in the group treated with $0.75 \mathrm{mg} / \mathrm{kg}$ and these included weight gain, Cushingoid appearance, and excessive hair growth. This study confirmed previous reports from these Centers showing that Prednisone produces a significant increase in muscle strength, pulmonary function, and functional ability in patients with Duchenne dystrophy. The present study also defines the minimu Prednisone dose required to produce the maximal increase in strength at 0.3 to $0.75 \mathrm{mg} / \mathrm{kg}$. Improvement is rapid occurring within ten days and is maximal by two months of treatment. The authors recommend that a Prednisone dosage of $0.75 \mathrm{mg} / \mathrm{kg} /$ day be considered for patients with Duchenne dystrophy who experience functional decline. (Griggs RC et al. Prednisone in Duchenne dystrophy. A randamized, controlled trial defining the time course and dose response. Arch Neurol April 1991; 48:383-388).

COMMENT. Clinical trials of myoblast transfer are investigational and limited in application. Studies of the benefits and mechanism of Prednisone in muscle protein synthesis are important particularly in young children showing a rapid functional decline. Alternative schedules of Prednisone administration such as pulse dosage are under investigation and may have fewer side effects. The beneficial effects of prednisone, 0.65 $\mathrm{mg} / \mathrm{kg} /$ day, extending over a two year observation period has been demonstrated in 89 boys with IMD. (Fenichel GM et al. Neurology March 1991; 41 (Suppl 1):166). 